\title{
Relationship among Sleep Quality Physical Health Conditions and Lifestyle Habits among Elementary School Students
}

\author{
Hiroko Sugimoto', Tetsuya Tanioka², Yuko Yasuhara², Kenji Mori², Yoshiko Gogi ${ }^{3}$, \\ Hiroki Mori ${ }^{3}$, Mihoko Nakanii ${ }^{4}$, Rozzano Locsin ${ }^{2}$ \\ ${ }^{1}$ Graduate School of Health Sciences, Tokushima University, Tokushima, Japan \\ ${ }^{2}$ Institute of Biomedical Sciences, Tokushima University, Tokushima, Japan \\ ${ }^{3}$ Department of Educational Psychology, Shujitsu University, Okayama, Japan \\ ${ }^{4}$ Faculty of Medical Welfare, Kawasaki University of Medical Welfare, Okayama, Japan \\ Email: hirokos1014@outlook.jp
}

How to cite this paper: Sugimoto, H., Tanioka, T., Yasuhara, Y., Mori, K., Gogi, Y., Mori, H., Nakanii, M. and Locsin, R. (2017) Relationship among Sleep Quality Physical Health Conditions and Lifestyle Habits among Elementary School Students. Open Journal of Psychiatry, 7, 235-247.

https://doi.org/10.4236/ojpsych.2017.74021

Received: May 31, 2017

Accepted: August 13, 2017

Published: August 16, 2017

Copyright $\odot 2017$ by authors and Scientific Research Publishing Inc. This work is licensed under the Creative Commons Attribution International License (CC BY 4.0).

http://creativecommons.org/licenses/by/4.0/

\begin{abstract}
The purpose of this research was to reveal the relationship among students' sleep quality, body temperature and lifestyle habits, growth and their physical strength. Subjects were 226 elementary school students from first grade to sixth grade, in four elementary schools at the Chugoku and Shikoku areas in Japan. The study period was from October 2014 to December 2015. Evaluation items used were the PSQI (Pittsburgh Sleep Quality Index, Japanese version), body temperature, lifestyle habits, and national physical fitness test. Relationships were analyzed using Pearson's chi square test, Fisher's exact test, residual analysis, $\varphi$-coefficient, odds ratio and $95 \%$ confidence interval, and Spearman's rank correlation coefficient using SPSS 20 (SPSS Inc., Chicago, IL, USA). The level of statistical significance was set at 0.05 . The number of students with good sleep quality who showed less than six-point score at the PSQI was 218 (96.5\%), and with poor sleep quality were eight students (3.5\%). Especially, "sometimes do not eat breakfast" and "had difficulty sleeping" were independent risk factors for negative arousal. In the lifestyle habits, it was observed significantly that students who had a good sleep ate breakfast every morning. The significant positive correlation was between sleep quality and the time spent watching television, the age and the time using the internet. The significant negative correlation was observed between length of sleep, time spent watching television, using internet and playing games, body temperature and age. The students with temperature of less than $36^{\circ} \mathrm{C}$ were 35 (15.5\%). Those students did not eat breakfast every morning, or ate school lunch. This study revealed that sleep condition and temperature of elementary
\end{abstract}


school students were related to eating breakfast and using media time. It was considered very important to educate the students and their guardians that eating breakfast is a necessity and adjusting the use of multimedia, so that students can acquire desirable lifestyle habits.

\section{Keywords}

Elementary Students, Lifestyle Habits, Multimedia Use, Sleep Quality, Body Temperature

\section{Introduction}

Sleep is important for the growth and development of student. A lack of sleep increases the risk of obesity [1], drowsiness during the daytime and careless behaviors [2] [3]. Furthermore, it is reported that lack of sleep decreases children's memory power, cognitive functions, and degrades their achievements [4] [5] [6] [7]. Those who go to bed before 10 p.m., and those who sleep more than eight hours a day, have a higher motivation to go to school [8]. It is also suggested that people who lack sleep and have difficulty sleeping tend to develop lifestyle habits that lead to disease [9] [10] [11]. Those who have insomnia symptoms tend to develop depression [12] show some physical complaints, and their motivation tends to decrease [13].

Worldwide, young people of Asian descent tend to go to bed later at night and have shorter sleeping hours during weekdays than their North American and European counterparts [14]. The Japanese, in particular, have shorter sleeping hours than those in other countries. According to the International Comparative Study by the Organisation for Economic Co-operation and Development (OECD), the comparison of sleeping hours of people from 28 countries in the world, aged from 15 to 64 , shows that Japanese sleeping hours are seven hours and 43 minutes, the shortest in the world [15]. According to the National Students Foundation, the sleeping hours required by school-age children are from 9 to 11 hours [16].

However, only $60 \%$ of Japanese primary school children get more than eight hours of sleep [17]. According to the International Comparative Study of the sleeping hours of infants' age three years or younger, Japanese infants were the shortest among 17 countries and regions [18]. As seen above, the Japanese have formed the habit of short sleeping hours since their infancy.

Meanwhile, the students-wake rhythm is influenced by body temperature fluctuations [19]. It is reported that the number of children who have low normal body temperatures has been increasing since around 1980 in Japan [20]. The reasons are as follows: Changes in lifestyle habits, such as the reduction of sleeping hours caused by late time of students; no breakfast intake; and the decline in the amount of exercise [21]. Also, it is shown that their low body temperature 
leads to the lowering of their motivation to go to school [22].

However, the relationship among sleep quality, low temperature, and physical health conditions of Japanese primary school children is still not clear. Understanding those relationship is considered to lead to an appropriate support to improve the factors that influence their lifestyle habits and low body temperature. Furthermore, promoting the health of children is essential to support toward the health of the next generation, and healthy lifestyles of children from primary school.

The purpose of this research was to reveal the relationship among elementary school students' sleep quality, body temperature and lifestyle habits, and physical strength.

\section{Methods}

The subjects were 226 students from first grade to sixth grade in four elementary schools at the Chugoku and Shikoku area, in Japan. The breakdown was 140 males (61.9\%), and 86 females (38.1\%). There were 29 first grade (12.8\%), 43 second grade (19.0\%), 39 third grade (17.3\%), 35 fourth grade (15.5\%), 48 fifth grade $(21.2 \%)$, and 32 sixth grade (14.2\%) students.

The study period was from October2014 to December 2015. The survey was conducted after permission was obtained from each of the school principals. Classroom teacher explained the reason and process of this survey. The purpose of this survey was explained to the guardians of the students, and a written permission form was sent to them that clearly stated that the results of the survey would not be used for purposes other than this research. Permission was obtained in writing thereby the students then answered the questionnaire about lifestyle habits.

Evaluation items were PSQI-J (Pittsburgh Sleep Quality Index, Japanese version), body temperatures, lifestyle habits (Breakfast, Bowel movement, School meal, Snack, Exercise, Cram school, Wake up, Falling asleep), and national physical fitness test.

PSQI assesses sleep quality during the previous month [23]. PSQI is composed of seven subscales, sleep quality, sleep latency, sleep duration, habitual sleep efficiency, sleep disturbance, use of sleeping medication, and daytime dysfunction. Total score is 0 to 21 point. Higher scores of 6 or more points represent poor subjective students [23] [24].

Body temperature was measured from 8:30 a.m. to 9:40 a.m. using Thermofocus ${ }^{\circledR}$ Pro, a non-contact infrared thermometer (Tecnimed Sri: Italy). Data over five days were obtained in order to estimate the mean value of body temperature detected from the skin surface of the forehead.

National physical fitness test results were compared using mean scores of physical tests obtained from all of Japan in 2014 and 2015 and evaluated as either high or low levels. Numbers and percentages were used to describe characteristics of subjects. The percentages were rounded up to two decimal points. The 
total may not equal $100 \%$.

The relationships were analyzed using Pearson's chi square test, Fisher's exact test, residual analysis, $\varphi$-coefficient, odds ratio and the $95 \%$ confidence interval, and Spearman's rank correlation coefficient. Some values for chi square test were calculated excluding missing values. Statistical analyses were performed using SPSS 20 (SPSS Inc., Chicago, IL, USA). The level of statistical significance was set at 0.05 .

\section{Results}

There were eight students (3.5\%) with poor sleep quality, whose PSQI scores were more than six points. The result of the analysis of the relationship between the sleep quality and lifestyle habits was not significant (Table 1).

Some subjects claimed, "I sometimes do not eat breakfast" (odds ratio [OR] = 5.20, 95\% confidence interval [CI]: 1.08 - 25.13) and "I had difficulty sleeping." $(\mathrm{OR}=42.58 .95 \% \mathrm{CI}: 4.80$ - 377.62), these were significant risk factor of negative arousal (Table 2).

Table 3 exhibits the findings relating of those students could fell asleep quickly and ate breakfast every morning $\left(\chi^{2}=11.13, \varphi=0.25, p<0.001\right)$.

The correlation among time spent using media, body temperature, sleep quality, length of sleep and age were shown in Table 4 . The significant positive correlation between the sleep quality and the time spent watching television (TV) ( $\rho$ $=0.13, p<0.05)$, the age and the time using internet $(\rho=0.30, p<0.001)$. The significant negative correlation was observed between among length of sleep, spent watching TV $(\rho=-0.28, p<0.001)$, using internet $(\rho=-0.15, p<0.05)$ and playing games $(\rho=-0.18, p<0.01)$, also body temperature and age $(\rho=$ $-0.17, p<0.05)$ in addition to length of sleep $(\rho=-0.37, p<0.001)$.

Average students' body temperature in five days less than $36^{\circ} \mathrm{C}$ was 35 (15.5\%), over $36^{\circ} \mathrm{C}$ was 191 (84.5\%). Students with body temperatures of less than $36^{\circ} \mathrm{C}$ were significantly related to the having eaten breakfast $\left(\chi^{2}=5.63, \varphi=\right.$ $-0.16, p<0.05)$, and eaten school meal ( $\left.\chi^{2}=8.46, \varphi=0.21, p<0.001\right)$ as compared with over $36^{\circ} \mathrm{C}$ body temperatures (Table 5 ).

\section{Discussion}

From the total scores of the PSQI, the number of students showing poor quality of sleep was eight, and the students showing uncomfortable arousal and those showing difficulty sleeping were twenty-four each.

Although there may have been differences in the results depending on whether or not the respondents to the questionnaire of lifestyle habits were guardians or students, it was clear that from this survey there were students who fit the condition of having difficulty sleeping.

When comfortable arousal was compared with lifestyle habits, it was revealed that the students who could fell asleep quickly woke up feeling refreshed, while those who ate a snack less than three days a week woke up feeling refreshed as 
Table 1. Comparison of the sleep quality and lifestyle habits.

\begin{tabular}{|c|c|c|c|c|c|c|c|c|c|c|}
\hline & & \multicolumn{6}{|c|}{ PSQI $(n=226)$} & \multirow{4}{*}{$X^{2}$ value } & \multirow{4}{*}{$\varphi$} & \multirow{4}{*}{$P$ value } \\
\hline & & \multirow{2}{*}{\multicolumn{3}{|c|}{$\begin{array}{l}\text { PSQI } \geq 6 \\
(n=8)\end{array}$}} & \multirow{2}{*}{\multicolumn{3}{|c|}{$\begin{array}{l}\text { PSQI }<6 \\
(\mathrm{n}=218)\end{array}$}} & & & \\
\hline & & & & & & & & & & \\
\hline & & $\mathrm{n}$ & $\%$ & $\begin{array}{l}\text { Adjusted } \\
\text { residual }\end{array}$ & $\mathrm{n}$ & $\%$ & $\begin{array}{l}\text { Adjusted } \\
\text { residual }\end{array}$ & & & \\
\hline \multicolumn{11}{|l|}{ Breakfast } \\
\hline$(\mathrm{n}=226)$ & $\begin{array}{l}\text { I eat breakfast every morning. } \\
\text { I sometimes do not eat breakfast. }\end{array}$ & 6 & $\begin{array}{l}2.65 \\
0.88\end{array}$ & $\begin{array}{c}-1.6 \\
1.6\end{array}$ & $\begin{array}{l}199 \\
19\end{array}$ & $\begin{array}{l}88.05 \\
8.41\end{array}$ & $\begin{array}{c}1.6 \\
-1.6\end{array}$ & 0.88 & -0.10 & 0.16 \\
\hline \multicolumn{11}{|c|}{ Bowel movement } \\
\hline$(\mathrm{n}=226)$ & $\begin{array}{l}\text { I have bowel movement every day. } \\
\text { I do not have bowel movement every day. }\end{array}$ & 4 & $\begin{array}{l}1.77 \\
1.77\end{array}$ & $\begin{array}{r}-0.5 \\
0.5\end{array}$ & $\begin{array}{l}130 \\
88\end{array}$ & $\begin{array}{l}57.52 \\
38.94\end{array}$ & $\begin{array}{l}0.5 \\
-0.5\end{array}$ & 0.03 & -0.04 & 0.72 \\
\hline $\begin{array}{l}\text { School me } \\
(\mathrm{n}=226)\end{array}$ & $\begin{array}{l}\text { I always eat a full school meal. } \\
\text { I sometimes leave part of a school meal. }\end{array}$ & 5 & $\begin{array}{l}2.21 \\
1.33\end{array}$ & $\begin{array}{l}0.0 \\
0.0\end{array}$ & $\begin{array}{l}136 \\
82\end{array}$ & $\begin{array}{l}60.18 \\
36.28\end{array}$ & $\begin{array}{l}0.0 \\
0.0\end{array}$ & 0.00 & 0.00 & 1.00 \\
\hline $\begin{array}{l}\text { Snack (lun } \\
(\mathrm{n}=226)\end{array}$ & $\begin{array}{l}\text { to dinner) } \\
\text { I eat a snack four days or more a week. } \\
\text { I eat a snack less than three days a week. }\end{array}$ & 4 & $\begin{array}{l}1.77 \\
1.77\end{array}$ & $\begin{array}{l}0.7 \\
-0.7\end{array}$ & $\begin{array}{l}82 \\
136\end{array}$ & $\begin{array}{l}36.28 \\
60.18\end{array}$ & $\begin{array}{c}-0.7 \\
0.7\end{array}$ & 0.11 & 0.05 & 0.48 \\
\hline $\begin{array}{l}\text { Snack }(\text { din } \\
(\mathrm{n}=226)\end{array}$ & $\begin{array}{l}\text { to fall asleep) } \\
\text { I eat a snack four days or more a week. } \\
\text { I eat a snack less than three days a week. }\end{array}$ & 7 & $\begin{array}{l}0.44 \\
3.10\end{array}$ & $\begin{array}{r}0.6 \\
-0.6\end{array}$ & $\begin{array}{r}15 \\
203\end{array}$ & $\begin{array}{r}6.64 \\
89.82\end{array}$ & $\begin{array}{c}-0.6 \\
0.6\end{array}$ & 0.00 & 0.04 & 0.45 \\
\hline$(n=226)$ & $\begin{array}{l}\text { I exercise three or more days a week. } \\
\text { I exercise less than two days a week. }\end{array}$ & $\begin{array}{l}6 \\
2\end{array}$ & $\begin{array}{l}2.65 \\
0.88\end{array}$ & $\begin{array}{c}-0.2 \\
0.2\end{array}$ & $\begin{array}{l}170 \\
48\end{array}$ & $\begin{array}{l}75.22 \\
21.24\end{array}$ & $\begin{array}{l}0.2 \\
-0.2\end{array}$ & 0.00 & -0.01 & 1.00 \\
\hline $\begin{array}{l}\text { Cram scho } \\
(\mathrm{n}=226)\end{array}$ & $\begin{array}{l}\text { I am going to cram school. } \\
\text { I am not going to cram school. }\end{array}$ & 5 & $\begin{array}{l}2.21 \\
1.33\end{array}$ & $\begin{array}{r}0.9 \\
-0.9\end{array}$ & $\begin{array}{l}101 \\
117\end{array}$ & $\begin{array}{l}44.69 \\
51.77\end{array}$ & $\begin{array}{c}-0.9 \\
0.9\end{array}$ & 0.29 & 0.06 & 0.48 \\
\hline $\begin{array}{l}\text { Physical st } \\
(\mathrm{n}=225)\end{array}$ & $\begin{array}{l}\text { gth } \\
\text { High } \\
\text { Low }\end{array}$ & 6 & $\begin{array}{l}0.89 \\
2.67\end{array}$ & $\begin{array}{r}1.6 \\
-1.6\end{array}$ & $\begin{array}{l}117 \\
100\end{array}$ & $\begin{array}{l}52.00 \\
44.44\end{array}$ & $\begin{array}{c}-1.6 \\
1.6\end{array}$ & 1.56 & 0.11 & 0.15 \\
\hline Falling asle & & & & & & & & & & \\
\hline$(\mathrm{n}=177)$ & $\begin{array}{l}\text { I could fall asleep quickly. } \\
\text { I had difficulty sleeping. }\end{array}$ & 2 & $\begin{array}{l}0.56 \\
1.13\end{array}$ & $\begin{array}{r}-2.7 \\
2.7\end{array}$ & $\begin{array}{l}152 \\
22\end{array}$ & $\begin{array}{l}85.88 \\
12.43\end{array}$ & $\begin{array}{l}2.7 \\
-2.7\end{array}$ & 3.46 & -0.20 & 0.05 \\
\hline$(\mathrm{n}=105)$ & $\begin{array}{l}\text { I woke up feeling refreshed. } \\
\text { I was sleepy. }\end{array}$ & $\begin{array}{l}2 \\
3\end{array}$ & $\begin{array}{l}1.90 \\
2.86\end{array}$ & $\begin{array}{l}-2.0 \\
2.0\end{array}$ & $\begin{array}{l}79 \\
21\end{array}$ & 75.24 & $\begin{array}{l}2.0 \\
-2.0\end{array}$ & 2.19 & -0.20 & 0.08 \\
\hline
\end{tabular}

Fisher's exact test. PSQI: Pittsburgh Sleep Quality Index. ${ }^{\star}$ Calculated by excluding "I was vaguely remember" from the response to "Falling asleep." ${ }^{*}$ Calculated by excluding "I was a little sleepy" from the response to "Wake up". 
Table 2. Comparison of wake up condition and lifestyle habits.

\begin{tabular}{|c|c|c|c|c|c|c|c|c|c|c|}
\hline & & \multicolumn{6}{|c|}{ Wake up $(\mathrm{n}=105)^{\star *}$} & \multirow{4}{*}{$\begin{array}{l}\text { Odds } \\
\text { Ratio }\end{array}$} & \multirow{3}{*}{\multicolumn{2}{|c|}{$\begin{array}{c}95 \% \\
\text { Confidence } \\
\text { Interval }\end{array}$}} \\
\hline & & \multirow{2}{*}{\multicolumn{3}{|c|}{$\begin{array}{l}\text { I woke up feeling refreshed. } \\
\qquad(\mathrm{n}=81)\end{array}$}} & \multirow{2}{*}{\multicolumn{3}{|c|}{$\begin{array}{l}\text { I was sleepy. } \\
\qquad(\mathrm{n}=24)\end{array}$}} & & & \\
\hline & & & & & & & & & & \\
\hline & & $\mathrm{n}$ & $\%$ & $\begin{array}{c}\text { Adjusted } \\
\text { residual }\end{array}$ & $\mathrm{n}$ & $\%$ & $\begin{array}{c}\text { Adjusted } \\
\text { residual }\end{array}$ & & & \\
\hline \multicolumn{11}{|l|}{ Breakfast } \\
\hline \multirow{2}{*}{$(\mathrm{n}=105)$} & I eat breakfast every morning. & 78 & 74.29 & 2.2 & 20 & 19.05 & -2.2 & 5.20 & 1.08 & 25.13 \\
\hline & I sometimes do not eat breakfast. & 3 & 2.86 & -2.2 & 4 & 3.81 & 2.2 & & & \\
\hline \multicolumn{11}{|c|}{ Bowel movement } \\
\hline \multirow{2}{*}{$(\mathrm{n}=105)$} & I have bowel movement every day. & 51 & 48.57 & 0.4 & 14 & 13.33 & -0.4 & 1.21 & 0.48 & 3.07 \\
\hline & I do not have bowel movement every day. & 30 & 28.57 & -0.4 & 10 & 9.52 & 0.4 & & & \\
\hline \multicolumn{11}{|c|}{ School meal } \\
\hline \multirow{2}{*}{$(\mathrm{n}=105)$} & I always eat a full school meal. & 57 & 54.29 & 0.7 & 15 & 14.29 & -0.7 & 1.43 & 0.55 & 3.70 \\
\hline & I sometimes leave part of a school meal. & 24 & 22.86 & -0.7 & 9 & 8.57 & 0.7 & & & \\
\hline \multicolumn{11}{|c|}{ Snack (lunch to dinner) } \\
\hline \multirow{2}{*}{$(\mathrm{n}=105)$} & I eat a snack four days or more a week. & 26 & 24.76 & -2.3 & 14 & 13.33 & 2.3 & 0.34 & 0.13 & 0.86 \\
\hline & I eat a snack less than three days a week. & 55 & 52.38 & 2.3 & 10 & 9.52 & -2.3 & & & \\
\hline \multicolumn{11}{|c|}{ Snack (dinner to fall asleep) } \\
\hline \multirow{2}{*}{$(\mathrm{n}=105)$} & I eat a snack four days or more a week. & 4 & 3.81 & -0.6 & 2 & 1.90 & 0.6 & 0.57 & 0.10 & 3.33 \\
\hline & I eat a snack less than three days a week. & 77 & 73.33 & 0.6 & 22 & 20.95 & -0.6 & & & \\
\hline \multicolumn{11}{|l|}{ Exercise } \\
\hline \multirow{2}{*}{$(\mathrm{n}=105)$} & I exercise three or more days a week. & 69 & 65.71 & 1.2 & 18 & 17.14 & -1.2 & 1.92 & 0.63 & 5.81 \\
\hline & I exercise less than two days a week. & 12 & 11.43 & -1.2 & 6 & 5.71 & 1.2 & & & \\
\hline \multicolumn{11}{|c|}{ Cram school } \\
\hline \multirow{2}{*}{$(\mathrm{n}=105)$} & I am going to cram school. & 42 & 40.00 & 0.5 & 11 & 10.48 & -0.5 & 1.27 & 0.51 & 3.17 \\
\hline & I am not going to cram school. & 39 & 37.14 & -0.5 & 13 & 12.38 & 0.5 & & & \\
\hline \multicolumn{11}{|c|}{ Physical strength } \\
\hline \multirow{2}{*}{$(\mathrm{n}=104)$} & High & 44 & 42.31 & 0.8 & 11 & 10.58 & -0.8 & 1.44 & 0.58 & 3.61 \\
\hline & Low & 36 & 34.62 & -0.8 & 13 & 12.50 & 0.8 & & & \\
\hline \multicolumn{11}{|c|}{ Falling asleep ${ }^{\star}$} \\
\hline \multirow{2}{*}{$(\mathrm{n}=93)$} & I could fall asleep quickly. & 73 & 78.49 & 3.3 & 12 & 12.90 & -3.3 & 42.58 & 4.80 & 377.62 \\
\hline & I had difficulty sleeping. & 1 & 1.08 & -3.3 & 7 & 7.53 & 3.3 & & & \\
\hline
\end{tabular}

*Calculated by excluding "I was vaguely remember" from the response to "Falling asleep." ${ }^{*}$ Calculated by excluding "I was a little sleepy" from the response to "Wake up". 
Table 3. Comparison of falling asleep and lifestyle habits.

\begin{tabular}{|c|c|c|c|c|c|c|c|c|c|c|}
\hline & & \multicolumn{6}{|c|}{ Falling asleep $(n=177)^{*}$} & \multirow{4}{*}{$\mathrm{X}^{2}$ value } & \multirow{4}{*}{$\varphi$} & \multirow{4}{*}{$P$ value } \\
\hline & & \multirow{2}{*}{\multicolumn{3}{|c|}{$\begin{array}{l}\text { I could fall asleep quickly. } \\
(\mathrm{n}=153)\end{array}$}} & \multirow{2}{*}{\multicolumn{3}{|c|}{$\begin{array}{l}\text { I had difficulty sleeping. } \\
\qquad(\mathrm{n}=24)\end{array}$}} & & & \\
\hline & & & & & & & & & & \\
\hline & & $\mathrm{n}$ & $\%$ & $\begin{array}{l}\text { Adjusted } \\
\text { residual }\end{array}$ & $\mathrm{n}$ & $\%$ & $\begin{array}{c}\text { Adjusted } \\
\text { residual }\end{array}$ & & & \\
\hline \multicolumn{11}{|l|}{ Breakfast } \\
\hline \multirow{2}{*}{$(\mathrm{n}=177)$} & I eat breakfast every morning. & 145 & 81.92 & 3.3 & 18 & 10.17 & -3.3 & 11.13 & 0.25 & $0.00^{* * *}$ \\
\hline & I sometimes do not eat breakfast. & 8 & 4.52 & -3.3 & 6 & 3.39 & 3.3 & & & \\
\hline \multicolumn{11}{|c|}{ Bowel movement } \\
\hline \multirow{2}{*}{$(\mathrm{n}=177)$} & I have bowel movement every day. & 100 & 56.50 & 1.5 & 12 & 6.78 & -1.5 & 2.11 & 0.11 & 0.15 \\
\hline & I do not have bowel movement every day. & 53 & 29.94 & -1.5 & 12 & 6.78 & 1.5 & & & \\
\hline \multicolumn{11}{|c|}{ School meal } \\
\hline \multirow{2}{*}{$(\mathrm{n}=177)$} & I always eat a full school meal. & 96 & 54.24 & 0.8 & 13 & 7.34 & -0.8 & 0.65 & 0.06 & 0.42 \\
\hline & I sometimes leave part of a school meal. & 57 & 32.20 & -0.8 & 11 & 6.21 & 0.8 & & & \\
\hline \multicolumn{11}{|c|}{ Snack (lunch to dinner) } \\
\hline \multirow{2}{*}{$(\mathrm{n}=177)$} & I eat a snack four days or more a week. & 61 & 34.46 & 0.2 & 9 & 5.08 & -0.2 & 0.05 & 0.02 & 0.83 \\
\hline & I eat a snack less than three days a week. & 92 & 51.98 & -0.2 & 15 & 8.47 & 0.2 & & & \\
\hline \multicolumn{11}{|c|}{ Snack (dinner to fall asleep) } \\
\hline \multirow{2}{*}{$(\mathrm{n}=177)$} & I eat a snack four days or more a week. & 10 & 5.65 & -1.0 & 3 & 3.00 & 1.0 & 0.39 & -0.08 & $0.39^{\mathrm{a})}$ \\
\hline & I eat a snack less than three days a week. & 143 & 80.79 & 1.0 & 21 & 11.86 & -1.0 & & & \\
\hline \multicolumn{11}{|l|}{ Exercise } \\
\hline \multirow{2}{*}{$(\mathrm{n}=177)$} & I exercise three or more days a week. & 126 & 71.19 & 1.8 & 16 & 9.04 & -1.8 & 3.22 & 0.14 & 0.07 \\
\hline & I exercise less than two days a week. & 27 & 15.25 & -1.8 & 8 & 4.52 & 1.8 & & & \\
\hline \multicolumn{11}{|c|}{ Cram school } \\
\hline \multirow{2}{*}{$(\mathrm{n}=177)$} & I am going to cram school. & 126 & 71.19 & 1.2 & 16 & 9.04 & -1.2 & 1.51 & 0.09 & 0.22 \\
\hline & I am not going to cram school. & 27 & 15.25 & -1.2 & 8 & 4.52 & 1.2 & & & \\
\hline \multicolumn{11}{|c|}{ Physical strength } \\
\hline \multirow{2}{*}{$(\mathrm{n}=176)$} & High & 90 & 51.14 & 1.2 & 11 & 6.25 & -1.2 & 1.52 & 0.09 & 0.22 \\
\hline & Low & 62 & 35.23 & -1.2 & 13 & 7.39 & 1.2 & & & \\
\hline
\end{tabular}

Pearson's chi-squared test. ${ }^{\text {a) }}$ Fisher's exact test; ${ }^{* * *}: p<0.001 .{ }^{*}$ Calculated by excluding "I was vaguely remember" from the response to "Falling asleep."

Table 4. Correlation among time spent using media, body temperature, sleep quality, length of sleep, and age.

\begin{tabular}{lcccccc}
\hline & \multicolumn{5}{c}{$\mathrm{n}=226$} \\
\cline { 2 - 7 } & $\begin{array}{c}\text { Sleep quality } \\
\text { (points) }\end{array}$ & $\begin{array}{c}\text { Watching TV } \\
\text { (hour) }\end{array}$ & $\begin{array}{c}\text { Using internet } \\
\text { (hour) }\end{array}$ & $\begin{array}{c}\text { Playing game } \\
\text { (hour) }\end{array}$ & $\begin{array}{c}\text { Length of sleep } \\
\text { (hour) }\end{array}$ & $\begin{array}{c}\text { Age } \\
\text { (years) }\end{array}$ \\
\hline Body temperature & $0.03^{\text {n.s. }}$ & $0.07^{\text {n.s. }}$ & $-0.01^{\text {n.s. }}$ & $-0.11^{\text {n.s. }}$ & $0.04^{\text {n.s. }}$ & $-0.17^{*}$ \\
Sleep quality & - & $0.13^{*}$ & $0.03^{\text {n.s. }}$ & $0.12^{\text {n.s. }}$ & $-0.10^{\text {n.s. }}$ & $-0.07^{\text {n.s. }}$ \\
Watching TV & - & - & $-0.02^{\text {n.s. }}$ & $0.22^{* *}$ & $-0.28^{* * *}$ & $0.08^{\text {n.s. }}$ \\
Using internet & & & - & $0.25^{* * *}$ & $-0.15^{*}$ & $0.30^{* * *}$ \\
Playing game & & & & - & $-0.18^{* *}$ & $0.13^{*}$ \\
Length of sleep & & & & & - & $-0.37^{* * *}$ \\
\hline
\end{tabular}

Spearman's rank correlation coefficient, n.s: not significant, ${ }^{*}: p<0.05,{ }^{* *}: p<0.01,{ }^{* * *}: p<0.001$. 
Table 5. Comparison of body temperature and lifestyle habits.

\begin{tabular}{|c|c|c|c|c|c|c|c|c|c|c|}
\hline & & & & Body temp & eratur & & & & & \\
\hline & & & $\begin{array}{l}\text { ody ten } \\
(\mathrm{n}=3\end{array}$ & $\begin{array}{l}\text { perature } \\
\text { 5) }\end{array}$ & Nor & $\begin{array}{l}\text { mal tem } \\
(\mathrm{n}=1\end{array}$ & $\begin{array}{l}\text { perature } \\
\text { 91) }\end{array}$ & $\begin{array}{c}X^{2} \\
\text { value }\end{array}$ & $\varphi$ & $P$ value \\
\hline & & $\mathrm{n}$ & $\%$ & $\begin{array}{c}\text { Adjusted } \\
\text { residual }\end{array}$ & $\mathrm{n}$ & $\%$ & $\begin{array}{c}\text { Adjusted } \\
\text { residual }\end{array}$ & & & \\
\hline Breakfast & & & & & & & & & & \\
\hline$(n-226)$ & I eat breakfast every morning. & 28 & 12.39 & -2.4 & 177 & 78.32 & 2.4 & 5.63 & -0.16 & $0.02^{*}$ \\
\hline$(11-220)$ & I sometimes do not eat breakfast. & 7 & 3.10 & 2.4 & 14 & 6.19 & -2.4 & & & \\
\hline Bowel mo & & & & & & & & & & \\
\hline$(n-226)$ & I have bowel movement every day. & 18 & 7.96 & -1.0 & 116 & 51.33 & 1.0 & 1.06 & -0.07 & 0.30 \\
\hline 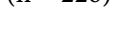 & I do not have bowel movement every day. & 17 & 7.52 & 1.0 & 75 & 33.19 & 1.0 & & & \\
\hline School me & & & & & & & & & & \\
\hline$(\mathrm{n}=226)$ & I always eat a full school meal. & 30 & 13.27 & 3.1 & 111 & 49.12 & -3.1 & 8.46 & 0.21 & $0.00^{\mathrm{a}) * * *}$ \\
\hline (114 - & I sometimes leave part of a school meal. & 5 & 2.21 & -3.1 & 80 & 35.40 & 3.1 & & & \\
\hline Snack (lun & iner) & & & & & & & & & \\
\hline$(n=226)$ & I eat a snack four days or more a week. & 13 & 5.75 & -0.1 & 73 & 32.30 & 0.1 & 0.02 & -0.01 & 0.90 \\
\hline & I eat a snack less than three days a week. & 22 & 9.73 & 0.1 & 118 & 52.21 & -0.1 & & & \\
\hline Snack (din & 11 asleep) & & & & & & & & & \\
\hline$(n=226)$ & I eat a snack four days or more a week. & 1 & 0.44 & -1.1 & 15 & 6.64 & 1.1 & 0.49 & -0.07 & $0.48^{\mathrm{a})}$ \\
\hline$(11-220)$ & I eat a snack less than three days a week. & 34 & 15.04 & 1.1 & 176 & 77.88 & -1.1 & & & \\
\hline Exercise & & & & & & & & & & \\
\hline$(n=226)$ & I exercise three or more days a week. & 26 & 11.50 & -0.6 & 150 & 66.37 & 0.6 & 0.31 & -0.04 & 0.58 \\
\hline & I exercise less than two days a week. & 9 & 3.98 & 0.6 & 41 & 18.14 & -0.6 & & & \\
\hline Cram scho & & & & & & & & & & \\
\hline$(\mathrm{n}=226)$ & I am going to cram school. & 16 & 7.08 & -0.2 & 90 & 39.82 & 0.2 & 0.02 & -0.01 & 0.88 \\
\hline & I am not going to cram school. & 19 & 8.41 & 0.2 & 101 & 44.69 & -0.2 & & & \\
\hline PSQI & & & & & & & & & & \\
\hline$(n-226)$ & $\mathrm{PSQI} \geq 6$ & 2 & 0.88 & -0.8 & 6 & 2.65 & 0.8 & 0.07 & -0.05 & $0.36^{\mathrm{a})}$ \\
\hline$(11+-220)$ & PSQI $<6$ & 33 & 14.60 & 0.8 & 185 & 81.86 & -0.8 & & & \\
\hline Physical st & & & & & & & & & & \\
\hline$(n=225)$ & High & 17 & 7.56 & 0.4 & 102 & 45.33 & -0.4 & 0.13 & 0.02 & 0.71 \\
\hline & Low & 17 & 7.56 & -0.4 & 89 & 39.56 & 0.4 & & & \\
\hline Falling asle & & & & & & & & & & \\
\hline$(\mathrm{n}=177)$ & I could fall asleep quickly. & 19 & 10.73 & 0.0 & 134 & 75.71 & 0.0 & 0.00 & 0.00 & $1.00^{\mathrm{a})}$ \\
\hline & I had difficulty sleeping. & 3 & 1.69 & 0.0 & 21 & 11.86 & 0.0 & & & \\
\hline Wake up ${ }^{\# \#}$ & & & & & & & & & & \\
\hline$(n-105)$ & I woke up feeling refreshed. & 12 & 11.43 & 0.3 & 69 & 65.71 & -0.3 & 0.00 & 0.03 & $1.00^{\mathrm{a})}$ \\
\hline & I was sleepy. & 3 & 2.86 & -0.3 & 21 & 20.00 & 0.3 & & & \\
\hline
\end{tabular}

Pearson's chi-squared test. ${ }^{a)}$ Fisher's exact test; ${ }^{*}: p<0.05,{ }^{* * *}: p<0.001$. Low body temperature: body temperatures of less than $36^{\circ} \mathrm{C}$, Normal temperature: over $36^{\circ} \mathrm{C}$ body temperatures. "Calculated by excluding "I was vaguely remember" from the response to "Falling asleep." "\#alculated by excluding "I was a little sleepy" from the response to "Wake up". 
well. It was reported that short sleeping times increase appetite [25] [26]. However in this research, because it was not surveyed whether snacks were prepared at home or through the childcare providers that they were using, further surveys are required in the future, including the positive detailed family environment and habits of regular exercise.

Students who could fall asleep quickly tended to eat breakfast every morning. Those whose body temperature was normal tended to eat breakfast every morning, as compared with those whose body temperature was low. It was also reported that if breakfast was not eaten, body temperatures do not rise and intellectual working capacity [27] does not increase and that eating breakfast more often leads to better students-wake rhythm [28].

In our study, some students responded that "I sometimes do not eat breakfast" and "I had difficulty sleeping." These were independent risk factors for negative arousal.

According to a cross-sectional study targeting middle and high school students, the more often they did not eat breakfast, the more they tended to complain about having difficulty sleeping, nocturnal awakening, early-morning awaking and insomnia [29]. Therefore, it was considered important to eat breakfast in order to elevate their motivation of performing activities during the daytime, and improve sleep quality.

As seen from the relationships between sleep quality and lifestyle habits, the longer the time spent using TV and games, the worse the sleep quality; and the longer they spent time using TV, games and Internet, the shorter their sleeping time.

It was also verified that lack of students causes various disorders, causes the risk of the occurrence of various disorders in autonomic nerve activity [30] [31], the endocrine system [32]-[37] and the cardiovascular system [38]-[43]. Therefore, in order to prevent diseases in the future, it is important to acquire a desirable life rhythm and maintain enough students from a very young age.

It was reported that the habits of TV watching of elementary school students is related to the presence or non-presence of sleep disorders [44]. It is clarified that infant TV watching habits are connected to short sleep times [45]. Also, in this research, there was a result supporting this precedent study. Regarding the use of media, time spent using games was related to both TV and Internet, and it was supposed that playing game was the main purpose of using media. It is, therefore, important to limit the time spent using games and appropriately use media in order to secure sleeping time.

The study has several limitations. One of these is the small sample size which may limit the generalization derived from the findings. As survey sheet problems, some responses were excluded and calculated: "I was vaguely remember" from the response to "Falling asleep"; and "I was a little sleepy" from response to "Wake up". 


\section{Conclusion}

The number of students with a good sleep quality who show less than six points score at the PSQI was 218 (96.5\%), and the number of students poor sleep quality was $8(3.5 \%)$. In the lifestyle habits, it was observed significantly good sleep quality and the students who eat breakfast every morning. The significant positive correlation between the sleep quality and the time spent watching TV, the age and the time using internet. The significant negative correlation was observed among length of sleep, spent watching TV, using internet and playing games, body temperature and age in addition to length of sleep. The number of students with body temperature less than $36^{\circ} \mathrm{C}$ was 35 (15.5\%). Those students did not eat breakfast every morning, and eat school lunch all. Especially, "sometimes do not eat breakfast" and "had difficulty sleeping" were independent risk factors for negative arousal. This study revealed that sleep quality and body temperature of elementary school students were related to breakfast intake and using time of media; the sleep quality and body temperature were related to their breakfast intake and time spent using media. It was considered very important to educate toward the students and their guardians that necessity of breakfast intake and how to deal with multimedia so that they can acquire a desirable lifestyle habits. A more detailed survey on the amount of time media is used for, its kinds of media, devices, and its influence on students' mental conditions, physical conditions, and academic achievement should be implemented in the future.

\section{Acknowledgements}

We would like to express our deep gratitude to everyone who cooperated in this research, including students and guardians who cooperated with the survey in carrying out this research.

\section{Disclosure}

All of the co-authors declare that they have no direct conflict of interest or grant support that is directly related to the content of the study.

\section{References}

[1] Snell, E.K., Adam, E.K. and Duncan, G.J. (2007) Sleep and the Body Mass Index and Overweight Status of Children and Adolescents. Child Development, 78, 309-323. https://doi.org/10.1111/j.1467-8624.2007.00999.x

[2] Fallone, G., Acebo, C., Arnedt, J.T., et al. (2001) Effects of Acute Sleep Restriction on Behavior, Sustained Attention, and Response Inhibition in Children. Perceptual and Motor Skills, 93, 213-229. https://doi.org/10.2466/pms.2001.93.1.213

[3] Sadeh, A., Gruber, R. and Raviv, A. (2003) The Effects of Sleep Restriction and Extension on School-Age Children: What a Difference an Hour Makes. Child Development, 74, 444-455. https://doi.org/10.1111/1467-8624.7402008

[4] Steenari, M.R., Vuontela, V., Paavonen, E.J., et al. (2003) Working Memory and Sleep in 6- to 13-Year-Old Schoolchildren. Journal of the American Academy of Child and Adolescent Psychiatry, 42, 85-92.

https://doi.org/10.1097/00004583-200301000-00014 
[5] Kuula, L., Pesonen, A.K., Martikainen, S., et al. (2015) Poor Sleep and Neurocognitive Function in Early Adolescence. Sleep Medicine, 16, 1207-1212. https://doi.org/10.1016/j.sleep.2015.06.017

[6] Medeiros, A.L.D., Mendes, D.B.F., Lima, P.F., et al. (2001) The Relationships between Sleep-Wake Cycle and Academic Performance in Medical Sleep. Journal Biological Rhythm Research, 32, 263-270. https://doi.org/10.1076/brhm.32.2.263.1359

[7] Ratcliff, R. and Van Dongen, H.P. (2009) Sleep Deprivation Affects Multiple Distinct Cognitive Processes. Psychonomic Bulletin \& Review, 16, 742-751. https://doi.org/10.3758/PBR.16.4.742

[8] Kadota, M., Yoshida, H., Daito, S., et al. (2012) The Life Style that Has Influenced the Japanese Elementary School Sixth-Grade Children's Motivation to Attend School. The Japan Society of Health Sciences of Mind and Body, 8, 150-159.

[9] Gangwisch, J.E., Heymsfield, S.B., Boden-Albala, B., et al. (2006) Short Sleep Duration as a Risk Factor for Hypertension: Analyses of the First National Health and Nutrition Examination Survey. Hypertension, 47, 833-839. https://doi.org/10.1161/01.HYP.0000217362.34748.e0

[10] James, E.G., Steven, B.H., Bernadette, B.A., et al. (2007) Sleep Duration as a Risk Factor for Diabetes Incidence in a Large US Sample. Sleep, 30, 1667-1673. https://doi.org/10.1093/sleep/30.12.1667

[11] Qureshi, A.I., Giles, W.H., Croft, J.B., et al. (1997) Habitual Sleep Patterns and Risk for Stroke and Coronary Heart Disease: A 10-Year Follow-Up from NHANES I. Neurology, 48, 904-911. https://doi.org/10.1212/WNL.48.4.904

[12] Buysse, D.J., Angst, J. and Gamma, A. (2008) Prevalence, Course, and Comorbidity of Insomnia and Depression in Young Adults. Sleep, 31, 473-480. https://doi.org/10.1093/sleep/31.4.473

[13] Sarsour K., Van Brunt, D.L. and Johnston, J.A. (2010) Associations of Nonrestorative Sleep with Insomnia, Depression, and Daytime Function. Sleep Mecicine, 11, 965-972. https://doi.org/10.1016/j.sleep.2010.08.007

[14] Gradisar, M., Gardner, G. and Dohnt, H. (2011) Recent Worldwide Sleep Patterns and Problems during Adolescence: A Review and Meta-Analysis of Age, Region, and Sleep. Sleep Medicine, 12, 110-118. https://doi.org/10.1016/j.sleep.2010.11.008

[15] OECD DATA (2011) Balancing Paid Work, Unpaid Work and Leisure. http://www.oecd.org/gender/data/balancingpaidworkunpaidworkandleisure.htm

[16] National Sleep Foundation (2015) How Much Sleep Do We Really Need? https://sleepfoundation.org/how-sleep-works/how-much-sleep-do-we-really-need

[17] Ministry of Education, Culture Sports, Science and Technology-Japan. http://www.mext.go.jp/a_menu/sports/kodomo/zencyo/1364874.htm

[18] Mindell, J.A., Sadeh, A., Wiegand, B., et al. (2010) Cross-Cultural Differences in Infant and Toddler Sleep. Sleep Medicine, 11, 274-280. https://doi.org/10.1016/j.sleep.2009.04.012

[19] Nakazawa, Y. (1991) The Biological Rhythm and Sleep. Otologia Fukuoka, 37, 14191425.

[20] Kobayashi, I., Hirayama, M., Nanbu, H., et al. (1982) A Study on the Temperature of Children Part 1, The Normal Temperature of Children of the Times. Journal of Child Health, 41, 419-427.

[21] Takasaki, Y. (1997) Body Temperature in Modern Japanese Children. Japanese Journal Physiological Authropology, 2, 3-8. 
[22] Noi, S., Ozawa, H. and Masaki, T. (2003) Characteristics of Low Body Temperature in Secondary School Boys. International Journal of Sport and Health Science, 1, 182187. https://doi.org/10.5432/ijshs.1.182

[23] Buysse, D.J., Reynolds 3rd, C.F., Monk, T.H., et al. (1989) The Pittsburgh Sleep Quality Index: A New Instrument for Psychiatric Practice and Research. Psychiatry Research, 28, 193-213. https://doi.org/10.1016/0165-1781(89)90047-4

[24] Doi, Y., Minowa, M., Uchiyama, M., Okawa, M., et al. (2000) Psychometric Assessment of Subjective Sleep Quality Using the Japanese Version of the Pittsburgh Sleep Quality Index (PSQI-J) in Psychiatric Disordered and Control Subjects. Psychiatry Research, 97, 165-172. https://doi.org/10.1016/S0165-1781(00)00232-8

[25] Spiegel, K., Tasali, E., Penev, P., et al. (2004) Brief Communication: Sleep Curtailment in Healthy Young Men Is Associated with Decreased Leptin Levels, Elevated Ghrelin Levels, and Increased Hunger and Appetite. Internal Medicine, 141, 846850. https://doi.org/10.7326/0003-4819-141-11-200412070-00008

[26] Taheri, S., Lin, L., Austin, D., et al. (2004) Short Sleep Duration Is Associated with Reduced Leptin, Elevated Ghrelin, and Increased Body Mass Index. PLoS Medicine, 1(3), e62. https://doi.org/10.1371/journal.pmed.0010062

[27] Benton, D. and Parker, P.Y. (1998) Breakfast, Blood Glucose, and Cognition. The American Journal of Clinical Nutrition, 67, 772-778.

[28] Yamaguchi, M., Uemura, H., Katsuura-Kamano, S., et al. (2013) Relationship of Dietary Factors and Habits with Sleep-Wake Regularity. Asia Pacific Journal of Clinical Nutrition, 22, 457-465.

[29] Kaneita, Y., Ohida, T., Osaki, Y., et al. (2006) Insomnia among Japanese Adolescents: A Nationwide Representative Survey. Sleep, 29, 1543-1550. https://doi.org/10.1093/sleep/29.12.1543

[30] Tochikubo, O., lkeda, A., Miyajima, E., et al. (1996) Effects of Insufficient Sleep on Blood Pressure Monitored by a New Multibiomedical Recorder. Hypertension. 27, 1318-1324. https://doi.org/10.1161/01.HYP.27.6.1318

[31] Spiegel, K., Leproult, R. and Van Cauter, E. (1999) Impact of Sleep Debt on Metabolic and Endocrine Function. Lancet, 354, 1435-1439. https://doi.org/10.1016/S0140-6736(99)01376-8

[32] Beihl, D.A., Liese, A.D. and Haffner, S.M. (2009) Sleep Duration as a Risk Factor for Incident Type 2 Diabetes in a Multiethnic Cohort. Annals of Epidemiology, 19, 351357. https://doi.org/10.1016/j.annepidem.2008.12.001

[33] Chaput, J.P., Despres, J.P., Bouchard, C., et al. (2009) Sleep Duration as a Risk Factor for the Development of Type 2 Diabetes or Impaired Glucose Tolerance: Analyses of the Quebec Family Study. Sleep Medicine, 10, 919-924. https://doi.org/10.1016/j.sleep.2008.09.016

[34] Gangwisch, J.E., Heymsfield, S.B., Boden-Albala, B., et al. (2007) Sleep Duration as a Risk Factor for Diabetes Incidence in a Large US Sample. Sleep, 30, 1667-1673. https://doi.org/10.1093/sleep/30.12.1667

[35] Mallon, L., Broman, J. and Hetta, J. (2005) High Incidence of Diabetes in Men with Sleep Complaints or Short Sleep Duration: A 12-Year Follow-Up Study of a Middle-Aged Population. Diabetes Care, 28, 2762-2767. https://doi.org/10.2337/diacare.28.11.2762

[36] Xu, Q., Song, Y. and Hollenbeck, A. (2010) Day Napping and Short Night Sleeping Are Associated with Higher Risk of Diabetes in Older Adults. Diabetes Care, 33, 7883. https://doi.org/10.2337/dc09-1143 
[37] Gottlieb, D.J., Punjabi, N.M. and Newman, A.B. (2005) Association of Sleep Time with Diabetes Mellitus and Impaired Glucose Tolerance. Archives of Internal Medicine, 165, 863-867. https://doi.org/10.1001/archinte.165.8.863

[38] Amagai, Y., Ishikawa, S. and Gotoh, T. (2010) Sleep Duration and Incidence of Cardiovascular Events in a Japanese Population: The Jichi Medical School Cohort Study. Journal of Epidemiology, 20, 106-110. https://doi.org/10.2188/jea.JE20090053

[39] Ayas, N.T., White, D.P., Manson, J.E., et al. (2003) A Prospective Study of Sleep Duration and Coronary Heart Disease in Women. Archives of Internal Medicine, 163, 205-209. https://doi.org/10.1001/archinte.163.2.205

[40] Burazeri, G., Gofin, J. and Kark, J.D. (2003) Over 8 Hours of Sleep-Marker of Increased Mortality in Mediterranean Population: Follow-Up Population Study. Croatian Medical Journal, 44, 193-198.

[41] Hamazaki, Y., Morikawa, Y., Nakamura, K., et al. (2011) The Effects of Sleep Duration on the Incidence of Cardiovascular Events among Middle-Aged Male Workers in Japan. Scandinavian Journal of Work, Environment \& Health, 37, 411-417. https://doi.org/10.5271/sjweh.3168

[42] Meisinger, C., Heier, M., Lowel, H., et al. (2007) Sleep Duration and Sleep Complaints and Risk of Myocardial Infarction in Middle-Aged Men and Women from the General Population: The MONICA/KORA Augsburg Cohort Study. Sleep, 30, 1121-1127. https://doi.org/10.1093/sleep/30.9.1121

[43] Shankar, A., Koh, W.P., Yuan, J.M., et al. (2008) Sleep Duration and Coronary Heart Disease Mortality among Chinese Adults in Singapore: A Population-Based Cohort Study. American Journal of Epidemiology, 168, 1367-1373. https://doi.org/10.1093/aje/kwn281

[44] Owens, J., Maxim, R., McGuinn, M., et al. (1999) Television-Viewing Habits and Sleep Disturbance in School Children. Pediatrics, 104, e27. https://doi.org/10.1542/peds.104.3.e27

[45] Nevarez, M.D., Rifas-Shiman, S.L., Kleinman, K.P., et al. (2010) Associations of Early Life Risk Factors with Infant Sleep Duration. Academic Pediatrics, 10, 187-193. https://doi.org/10.1016/j.acap.2010.01.007 